PROCEEDINGS OF THE

AMERICAN MATHEMATICAL SOCIETY

Volume 138, Number 1, January 2010, Pages 209-216

S 0002-9939(09)10002-3

Article electronically published on July 30, 2009

\title{
DOMINATED POLYNOMIALS ON INFINITE DIMENSIONAL SPACES
}

\author{
GERALDO BOTELHO, DANIEL PELLEGRINO, AND PILAR RUEDA
}

(Communicated by Nigel J. Kalton)

\begin{abstract}
The aim of this paper is to prove a stronger version of a conjecture posed earlier on the existence of nondominated scalar-valued $m$-homogeneous polynomials, $m \geq 3$, on arbitrary infinite dimensional Banach spaces.
\end{abstract}

\section{INTRODUCTION}

The theory of absolutely summing multilinear mappings and homogeneous polynomials between Banach spaces, which was first outlined by Pietsch [43, studies multilinear and polynomial generalizations of the very successful theory of absolutely summing linear operators. The theory has been developed by several authors, and among the advances obtained thus far we mention: Pietsch-type domination/factorization theorems ([11], Geiss [26], Pérez-García [38]), different types of absolutely summing multilinear mappings and polynomials (Achour and Mezrag 11, Carando and Dimant 15, Çaliskan and Pellegrino [14, Dimant 23, Pellegrino and Souza [36, Pérez-García [38]), Grothendieck-type theorems (Bombal et al. 4, Pérez-García and Villanueva [41]), coincidence/inclusion/composition theorems (Alencar and Matos [2], Botelho et al. [8], Pérez-García [39, 37, Popa 44]), connections with the geometry of Banach spaces ([5, Floret and Matos 25], Meléndez and Tonge [33, 35, Pérez-García [40]), interplay with other multi-ideals and polynomial ideals (Botelho et al. 77, 12, Cilia and Gutiérrez 17, Jarchow et al. 27, Matos [29]), estimates for absolutely summing norms (Aron et al. 3], [13, Choi et al. [16, Defant and Sevilla-Peris [21, Zalduendo [45]), extensions of the theory to more general nonlinear mappings (Junek, Matos and Pellegrino 28, Matos [30, 31, Matos and Pellegrino 32]).

Let us sketch some of the applications this theory has produced. The following results were obtained with the help of the theory of absolutely summing multilinear mappings: (i) for every $n \in \mathbb{N}$, a tensor norm of order $n$ constructed by PérezGarcía and Villanueva 41] is shown in Defant and Pérez-García 20 to preserve unconditionality for $\mathcal{L}_{p}$-spaces; (ii) Defant et al. [19] provides optimal estimates for the width of Bohr's strip for Dirichlet series in infinite dimensional Banach spaces;

Received by the editors February 18, 2009, and, in revised form, April 6, 2009.

2000 Mathematics Subject Classification. Primary 46G25, 46B20.

The first author was supported by CNPq Grants 620108/2008-8 and 306981/2008-4.

The second author was supported by INCT-Matemática, CNPq Grants 620108/2008-8, 308084/2006-3 and 471686/2008-5.

The third author was supported by Ministerio de Ciencia e Innovación MTM2008-03211/MTM.

(C)2009 American Mathematical Society Reverts to public domain 28 years from publication 
(iii) applications to quantum information theory are obtained by Pérez-García et al. 42, where it is proved that, contrary to the bipartite case, tripartite Bell inequalities can be unboundedly violated; (iv) in Jarchow et al. [27], the existence of Hahn-Banach-type extension theorems for multilinear forms is strongly connected to the structural properties of the underlying spaces.

One of the central notions of this theory is that of a dominated homogeneous polynomial. A continuous $m$-homogeneous polynomial $P$ from the Banach space $X$ to the Banach space $Y$ is $r$-dominated if $\left(P\left(x_{j}\right)\right)_{j=1}^{\infty}$ is $\frac{r}{m}$-summable in $Y$ whenever $\left(x_{j}\right)_{j=1}^{\infty}$ is weakly $r$-summable in $X$.

The following conjecture was posed in 9]:

Conjecture 1. There is no infinite dimensional Banach space $X$ such that for every $m \in \mathbb{N}$ and every $r \geq 1$, any continuous scalar-valued $m$-homogeneous polynomial on $X$ is $r$-dominated.

It is known that the conjecture holds true for Banach spaces with unconditional basis (see [9. Theorem 3.2]). The status of the problem was changed by the proof of a stronger result for multilinear forms: in Jarchow et al. [27, Lemma 5.4] (see also [10, Proposition 3.2]), it is shown that for every $m \geq 3$ and every $r \geq 1$, on every infinite dimensional Banach space, there exists a continuous $m$-linear form that fails to be $r$-dominated. Although not solving Conjecture 1 (to prove Conjecture 1 we need a symmetric non- $r$-dominated $m$-linear form), this result indicates that a result stronger than Conjecture 1 should be pursued for polynomials:

Conjecture 2. For every infinite dimensional Banach space $X$, every $m \geq 3$ and every $r \geq 1$, there is a continuous scalar-valued $m$-homogeneous polynomial on $X$ that fails to be r-dominated.

In Section 2 we solve Conjecture 2 (hence Conjecture 1) in the positive.

\section{Notation}

Throughout this paper, $n$ and $m$ are positive integers, and $X$ and $Y$ will stand for Banach spaces over $\mathbb{K}=\mathbb{R}$ or $\mathbb{C}$. The Banach space of all continuous $m$ homogeneous polynomials $P: X \longrightarrow Y$, with the sup norm, is denoted by $\mathcal{P}\left({ }^{m} X ; Y\right)$ $(\mathcal{L}(X ; Y)$ if $m=1)$. When $m=1$ and $Y=\mathbb{K}$, we write $X^{*}$ to denote the topological dual of $X$. The closed unit ball of $X$ is represented by $B_{X}$. For details on the theory of polynomials between Banach spaces, we refer to [24, 34].

Given $r \in[0, \infty)$, let $\ell_{r}(X)$ be the Banach $(r$-Banach if $0<r<1$ ) space of all absolutely $r$-summable sequences $\left(x_{j}\right)_{j=1}^{\infty}$ in $X$ with the norm $\left\|\left(x_{j}\right)_{j=1}^{\infty}\right\|_{r}=$ $\left(\sum_{j=1}^{\infty}\left\|x_{j}\right\|^{r}\right)^{1 / r}$. We denote by $\ell_{r}^{w}(X)$ the Banach $(r$-Banach if $0<r<1)$ space of all weakly $r$-summable sequences $\left(x_{j}\right)_{j=1}^{\infty}$ in $X$ with the norm $\left\|\left(x_{j}\right)_{j=1}^{\infty}\right\|_{w, r}=$ $\sup _{\varphi \in B_{X *}}\left\|\left(\varphi\left(x_{j}\right)\right)_{j=1}^{\infty}\right\|_{r}$.

Let $p, q>0$. An $m$-homogeneous polynomial $P \in \mathcal{P}\left({ }^{m} X ; Y\right)$ is absolutely $(p ; q)$ summing if $\left(P\left(x_{j}\right)\right)_{j=1}^{\infty} \in \ell_{p}(Y)$ whenever $\left(x_{j}\right)_{j=1}^{\infty} \in \ell_{q}^{w}(X)$. It is well known that $P$ is absolutely $(p ; q)$-summing if and only if there is a constant $C \geq 0$ such that

$$
\left(\sum_{j=1}^{n}\left\|P\left(x_{j}\right)\right\|^{p}\right)^{\frac{1}{p}} \leq C\left(\left\|\left(x_{j}\right)_{j=1}^{n}\right\|_{w, q}\right)^{m}
$$


for every $x_{1}, \ldots, x_{n} \in X$ and $n$ a positive integer. The infimum of such a $C$ is denoted by $\|P\|_{a s(p ; q)}$. The space of all absolutely $(p ; q)$-summing $m$-homogeneous polynomials from $X$ to $Y$ is denoted by $\mathcal{P}_{a s(p ; q)}\left({ }^{m} X ; Y\right)$, and $\|\cdot\|_{a s(p ; q)}$ is a complete norm $(p$-norm if $p<1)$ on $\mathcal{P}_{a s(p ; q)}\left({ }^{m} X ; Y\right)$.

An $m$-homogeneous polynomial $P \in \mathcal{P}\left({ }^{m} X ; Y\right)$ is said to be $r$-dominated if it is absolutely $\left(\frac{r}{m} ; r\right)$-summing. In this case we write $\mathcal{P}_{d, r}\left({ }^{m} X ; Y\right)$ and $\|\cdot\|_{d, r}$ instead of $\mathcal{P}_{a s\left(\frac{r}{m} ; r\right)}\left({ }^{m} X ; Y\right)$ and $\|\cdot\|_{a s\left(\frac{r}{m} ; r\right)}$. As usual we write $\mathcal{P}_{d, r}\left({ }^{m} X\right)$ and $\mathcal{P}\left({ }^{m} X\right)$ when $Y=\mathbb{K}$. The definition (and notation) of $r$-dominated multilinear mappings is analogous (for the notation just replace $\mathcal{P}$ by $\mathcal{L}$ ). The symbol $\mathcal{L}^{s}$ means that only symmetric multilinear mappings are considered. For details we refer to 9,27 .

\section{The Proof of Conjecture 2}

It is well-known that a homogeneous polynomial is $p$-dominated if and only if its associated symmetric multilinear map is also $p$-dominated (see [33, Theorem 6]). However, the multilinear and polynomial parts of our problem are not so tightly connected, as is made clear by the following example of a non- $p$-dominated multilinear form whose symmetrization is $p$-dominated: let

$$
T: \ell_{2} \times \ell_{2} \rightarrow \mathbb{K}, T(x, y)=\sum_{j=1}^{\infty} x_{j} y_{j+1}-\sum_{j=1}^{\infty} x_{j+1} y_{j} .
$$

Note that $T\left(e_{j}, e_{j+1}\right)=1$ for every $j$. So, $T$ fails to be $p$-dominated (regardless of the $p \geq 1$ ). On the other hand, the symmetrization of $T$ is zero. So, even knowing that the multilinear counterpart of the conjecture is true, it is in principle not clear that the same holds for polynomials.

To prove Conjecture 2 we need the following well-known results:

Lemma 2.1. (a) [6. Propositions 41(b) and 46(a)] If $\mathcal{P}_{d, r}\left({ }^{n} X ; Y\right)=\mathcal{P}\left({ }^{n} X ; Y\right)$, then $\mathcal{P}_{d, r}\left({ }^{m} X ; Y\right)=\mathcal{P}\left({ }^{m} X ; Y\right)$ for every $m \leq n$.

(b) (11, Proposition 3.4] and [22, Theorem 2.8]) $\mathcal{P}_{d, r}\left({ }^{n} X ; Y\right) \subseteq \mathcal{P}_{d, q}\left({ }^{n} X ; Y\right)$ if $r \leq q$.

The following result has its proof inspired by the proof of [27, Lemma 5.4]; its final form was kindly suggested by the referee.

Theorem 2.2. If $X$ is infinite dimensional and $\mathcal{P}_{d, 2}\left({ }^{2} X\right)=\mathcal{P}\left({ }^{2} X\right)$, then $\mathcal{P}_{d, r}\left({ }^{m} X\right)$ $\neq \mathcal{P}\left({ }^{m} X\right)$ for every $m \geq 3$ and every $r \geq 1$.

Proof. By Lemma 2.1(b) we may assume $r \geq 2$, and by Lemma 2.1(a) we just need to prove the case $m=3$. Since $X$ is infinite dimensional, by Dvoretzky's theorem there is a sequence $\left(E_{n}\right)_{n=1}^{\infty}$ of subspaces of $X$ such that $X / E_{n}$ is 2-isomorphic to $\ell_{2}^{n}$ for every $n$ (details can be found in the proof of [22, Proposition 19.17(b)]). So we can consider isomorphisms $j_{n}: X / E_{n} \longrightarrow \ell_{2}^{n}$ such that $\left\|j_{n}\right\| \leq 4$ and $\left\|j_{n}^{-1}\right\|=\frac{1}{2}$ for every $n$. Letting $\pi_{n}: X \longrightarrow X / E_{n}$ be the corresponding quotient maps and defining $q_{n}:=j_{n} \circ \pi_{n}$ we have that

$$
\left\|q_{n}\right\| \leq 4 \text { and } B_{\ell_{2}^{n}} \subseteq q_{n}\left(B_{X}\right) \text { for every } n .
$$

By assumption we know that $\mathcal{L}_{d, 2}^{s}\left({ }^{2} X\right)=\mathcal{L}^{s}\left({ }^{2} X\right)$, so by the open mapping theorem there exists $C>0$, depending only on $X$, such that $\|A\|_{d, 2} \leq C\|A\|$ for every $A \in \mathcal{L}^{s}\left({ }^{2} X\right)$. Given $n \in \mathbb{N}$, defining

$$
A: X \times X \longrightarrow \mathbb{K}, A(x, y)=\left\langle q_{n}(x), q_{n}(y)\right\rangle,
$$


we have that $A$ is a continuous symmetric (in the complex case we consider its symmetrization) bilinear form on $X$ and $\|A\| \leq 16$ because $\left\|q_{n}\right\| \leq 4$. Given $x_{1}, \ldots, x_{k} \in X$

$$
\begin{aligned}
\sum_{j=1}^{k}\left\|q_{n}\left(x_{j}\right)\right\|_{2}^{2} & =\sum_{j=1}^{k}\left\langle q_{n}\left(x_{j}\right), q_{n}\left(x_{j}\right)\right\rangle=\sum_{j=1}^{k}\left|A\left(x_{j}, x_{j}\right)\right| \\
& \leq\|A\|_{d, 2}\left\|\left(x_{j}\right)_{j=1}^{k}\right\|_{w, 2}^{2} \leq C\|A\|\left\|\left(x_{j}\right)_{j=1}^{k}\right\|_{w, 2}^{2} \\
& \leq 16 C\left\|\left(x_{j}\right)_{j=1}^{k}\right\|_{w, 2}^{2},
\end{aligned}
$$

showing that $\left\|q_{n}\right\|_{a s(2,2)} \leq 4 \sqrt{C}$ for every $n$.

For each positive integer $n$, consider the canonical embedding $i_{n}: \ell_{2}^{n} \longrightarrow \ell_{\infty}^{n}$. It is plain that $\left\|i_{n}\right\|=1$ for every $n$ and $\lim _{n \rightarrow \infty}\left\|i_{n}\right\|_{a s(r ; r)}=\infty$. Again, by Dvoretzky's theorem, there is an $n$-dimensional subspace $X_{n}$ of $X$ and an isomorphism $k_{n}: X_{n} \longrightarrow \ell_{2}^{n}$ such that $\left\|k_{n}\right\| \leq 1$ and $\left\|k_{n}^{-1}\right\| \leq 2$ (for every $n$ ). Since $\ell_{\infty}^{n}$ is an injective space, there is a norm-preserving extension $u_{n}$ of $i_{n} \circ k_{n}$ to the whole of $X$. Let us see that $\sup _{n}\left\|u_{n}\right\|_{a s(r ; r)}=\infty$. Given $M>0$, since $\sup _{n}\left\|i_{n}\right\|_{a s(r, r)}=\infty$, there exists $n$ such that $\left\|i_{n}\right\|_{a s(r, r)}>M$. Then, there exist $m \in \mathbb{N}$ and $x_{1}, \ldots, x_{m} \in \ell_{2}^{n}$ such that

$$
\left(\sum_{j=1}^{m}\left\|i_{n}\left(x_{j}\right)\right\|_{\infty}^{r}\right)^{1 / r}>M\left\|\left(x_{j}\right)_{j=1}^{m}\right\|_{w, r} .
$$

Since the restriction of $u_{n}$ to $X_{n}$ is $i_{n} \circ k_{n}$, for any $x \in \ell_{2}^{n}$ we have

$$
\left\|i_{n}(x)\right\|_{\infty}=\left\|i_{n} \circ k_{n}\left(k_{n}^{-1}(x)\right)\right\|_{\infty}=\left\|u_{n}\left(k_{n}^{-1}(x)\right)\right\|_{\infty} .
$$

From (11) it follows that

$$
\begin{aligned}
\left(\sum_{j=1}^{m}\left\|u_{n}\left(k_{n}^{-1}\left(x_{j}\right)\right)\right\|_{\infty}^{r}\right)^{1 / r} & >M\left\|\left(x_{j}\right)_{j=1}^{m}\right\|_{w, r} \\
& =\frac{M}{2} \sup _{\varphi \in 2 B_{\left(\ell_{2}^{n}\right)^{*}}}\left(\sum_{j=1}^{m}\left|\varphi\left(x_{j}\right)\right|^{r}\right)^{1 / r} \\
& \stackrel{(*)}{\geq} \sup _{\psi \in B_{\left(X_{n}\right)^{*}}}\left(\sum_{j=1}^{m}\left|\psi\left(k_{n}^{-1}\left(x_{j}\right)\right)\right|^{r}\right)^{1 / r} \\
& =\frac{M}{2}\left\|\left(k_{n}^{-1}\left(x_{j}\right)\right)_{j=1}^{m}\right\|_{w, r} .
\end{aligned}
$$

$(*) \psi \in B_{\left(X_{n}\right)^{*}} \Longrightarrow\left\|\psi \circ k_{n}^{-1}\right\| \leq 2 \Longrightarrow \psi \circ k_{n}^{-1} \in 2 B_{\left(\ell_{2}^{n}\right)^{*}}$.

Hence, $\sup _{n}\left\|u_{n}\right\|_{a s(r, r)}=\infty$. Following the steps of the proof of [27, Lemma 5.4], $\ell_{\infty}^{n}$ can be identified with the diagonal of $\ell_{2}^{n} \otimes_{\varepsilon} \ell_{2}^{n}$, so that $u_{n}$ can be regarded as a linear operator from $X$ to the diagonal of $\ell_{2}^{n} \otimes_{\varepsilon} \ell_{2}^{n}$ whose norm does not depend on n. From

$$
\ell_{2}^{n} \otimes_{\varepsilon} \ell_{2}^{n} \stackrel{(* *)}{=}\left(\left(\ell_{2}^{n}\right)^{*} \otimes_{\pi}\left(\ell_{2}^{n}\right)^{*}\right)^{*}=\left(\ell_{2}^{n} \otimes_{\pi} \ell_{2}^{n}\right)^{*}=\mathcal{L}\left({ }^{2} \ell_{2}^{n}\right)
$$


(for $(* *)$ see [18, Proposition 4.1(1)]), $u_{n}$ can now be regarded as a linear operator from $X$ to $\mathcal{L}\left({ }^{2} \ell_{2}^{n}\right)$ such that $u_{n}(x)$ is a symmetric bilinear form (elements of the diagonal of the tensor product are symmetric tensors, to which correspond symmetric bilinear forms) for every $x \in X$.

Consider the 3-linear form

$$
T_{n}: X \times X \times X \longrightarrow \mathbb{K}, T_{n}(x, y, z)=u_{n}(x)\left(q_{n}(y), q_{n}(z)\right),
$$

its symmetrization $T_{n}^{s} \in \mathcal{L}^{s}\left({ }^{3} X\right)$,

$$
T_{n}^{s}(x, y, z)=\frac{1}{3}\left[T_{n}(x, y, z)+T_{n}(y, x, z)+T_{n}(z, x, y)\right],
$$

and the linear operator $\widetilde{T}_{n}^{s}: X \longrightarrow \mathcal{L}\left({ }^{2} X\right)$ associated to $T_{n}^{s}$,

$$
\begin{aligned}
\widetilde{T}_{n}^{s}(x)(y, z) & =\frac{1}{3}\left[T_{n}(x, y, z)+T_{n}(y, x, z)+T_{n}(z, x, y)\right] \\
& =\frac{1}{3}\left[u_{n}(x)\left(q_{n}(y), q_{n}(z)\right)+u_{n}(y)\left(q_{n}(x), q_{n}(z)\right)+u_{n}(z)\left(q_{n}(x), q_{n}(y)\right)\right] .
\end{aligned}
$$

Write $3 \widetilde{T}_{n}^{s}=A_{n}+B_{n}+C_{n}$, where

$$
\begin{aligned}
& A_{n}(x)(y, z)=u_{n}(x)\left(q_{n}(y), q_{n}(z)\right), \\
& B_{n}(x)(y, z)=u_{n}(y)\left(q_{n}(x), q_{n}(z)\right), \text { and } \\
& C_{n}(x)(y, z)=u_{n}(z)\left(q_{n}(x), q_{n}(y)\right) .
\end{aligned}
$$

Given $x_{1}, \ldots, x_{k} \in X$,

$$
\begin{aligned}
\sum_{j=1}^{k}\left\|B_{n}\left(x_{j}\right)\right\|^{2} & =\sum_{j=1}^{k} \sup _{y, z \in B_{X}}\left|u_{n}(y)\left(q_{n}\left(x_{j}\right), q_{n}(z)\right)\right|^{2} \\
& \leq \sum_{j=1}^{k}\left\|u_{n}\right\|^{2}\left\|q_{n}\left(x_{j}\right)\right\|^{2}\left\|q_{n}\right\|^{2} \\
& \leq \sup _{n}\left\|u_{n}\right\|^{2} \sup _{n}\left\|q_{n}\right\|^{2}\left\|q_{n}\right\|_{a s(2,2)}^{2}\left\|\left(x_{j}\right)_{j=1}^{k}\right\|_{w, 2}^{2},
\end{aligned}
$$

proving that $B_{n}$ is 2 -summing (hence $r$-summing because $r \geq 2$ ) and its 2 -summing norm (hence its $r$-summing norm) is controlled by $16 \sqrt{C} \sup _{n}\left\|u_{n}\right\|$. It is clear that the same happens to $C_{n}$, so

$$
\sup _{n}\left\|B_{n}\right\|_{a s(r, r)}<\infty \text { and } \sup _{n}\left\|C_{n}\right\|_{a s(r, r)}<\infty .
$$

Let us prove that $\sup _{n}\left\|A_{n}\right\|_{a s(r, r)}=\infty$. Given $M^{\prime}>0$, from $\sup _{n}\left\|u_{n}\right\|_{a s(r, r)}=\infty$ there is an $n$ such that $\left\|u_{n}\right\|_{a s(r, r)}>M^{\prime}$. So, there are $m \in \mathbb{N}$ and $y_{1}, \ldots, y_{m} \in X$ such that

$$
\left(\sum_{j=1}^{m}\left\|u_{n}\left(y_{j}\right)\right\|^{r}\right)^{\frac{1}{r}}>M^{\prime}\left\|\left(y_{j}\right)_{j=1}^{m}\right\|_{w, r} .
$$

Using that $B_{\ell_{2}^{n}} \subseteq q_{n}\left(B_{X}\right)$, we have

$$
\begin{aligned}
\left\|u_{n}(x)\right\| & =\sup _{\lambda_{1}, \lambda_{2} \in B_{\ell_{2}^{n}}}\left|u_{n}(x)\left(\lambda_{1}, \lambda_{2}\right)\right| \\
& \leq \sup _{y, z \in B_{X}}\left|u_{n}(x)\left(q_{n}(y), q_{n}(z)\right)\right| \\
& =\sup _{y, z \in B_{X}}\left|A_{n}(x)(y, z)\right|=\left\|A_{n}(x)\right\|,
\end{aligned}
$$


for every $x \in X$. So,

$$
\left(\sum_{j=1}^{m}\left\|A_{n}\left(y_{j}\right)\right\|^{r}\right)^{\frac{1}{r}} \geq\left(\sum_{j=1}^{m}\left\|u_{n}\left(y_{j}\right)\right\|^{r}\right)^{\frac{1}{r}}>M^{\prime}\left\|\left(y_{j}\right)_{j=1}^{m}\right\|_{w, r},
$$

proving that $\left\|A_{n}\right\|_{a s(r, r)}>M^{\prime}$; hence $\sup _{n}\left\|A_{n}\right\|_{a s(r, r)}=\infty$. From $3 \widetilde{T}_{n}^{s}=A_{n}+$ $B_{n}+C_{n}$, it follows that $\sup _{n}\left\|\widetilde{T}_{n}^{s}\right\|_{a s(r, r)}=\infty$. Hence $\sup _{n}\left\|T_{n}^{s}\right\|_{d, r}=\infty$ because $\left\|\widetilde{T}_{n}^{s}\right\|_{a s(r, r)} \leq\left\|T_{n}^{s}\right\|_{d, r}$ (see, e.g., [5] Lemma 3.4]). But $\sup _{n}\left\|T_{n}^{s}\right\|<\infty$ because $\sup _{n}\left\|u_{n}\right\|<\infty$ and $\sup _{n}\left\|q_{n}\right\|<\infty$, so the open mapping theorem yields that $\mathcal{L}^{s}\left({ }^{3} X\right) \neq \mathcal{L}_{d, r}^{s}\left({ }^{3} X\right)$. Therefore $\mathcal{P}\left({ }^{3} X\right) \neq \mathcal{P}_{d, r}\left({ }^{3} X\right)$.

To complete the proof of Conjecture 2 we need another well-known result (for complex scalars the proof appeared in [25, Corollary 3.2]; the general case can be found in [17, Proposition 13]):

Lemma 2.3. $\mathcal{P}_{d, r}\left({ }^{2} X\right)=\mathcal{P}_{d, 2}\left({ }^{2} X\right)$ for every Banach space $X$ and $r \geq 2$.

Theorem 2.4. Let $m \geq 3, r \geq 1$ and $X$ be an infinite dimensional Banach space. Then

$$
\mathcal{P}_{d, r}\left({ }^{m} X\right) \neq \mathcal{P}\left({ }^{m} X\right) .
$$

Moreover, if $\mathcal{P}_{d, 2}\left({ }^{2} X\right) \neq \mathcal{P}\left({ }^{2} X\right)$, then (2) holds for every $m \geq 2$ and $r \geq 1$.

Proof. If $\mathcal{P}_{d, 2}\left({ }^{2} X\right)=\mathcal{P}\left({ }^{2} X\right)$, Theorem 2.2 gives the result. If $\mathcal{P}_{d, 2}\left({ }^{2} X\right) \neq \mathcal{P}\left({ }^{2} X\right)$, Lemma 2.3 gives that $\mathcal{P}_{d, r}\left({ }^{2} X\right) \neq \mathcal{P}\left({ }^{2} X\right)$ for every $r \geq 2$. Hence $\mathcal{P}_{d, r}\left({ }^{2} X\right) \neq \mathcal{P}\left({ }^{2} X\right)$ for every $r \geq 1$ by Lemma 2.1(b). Lemma 2.1(a) completes the result for every $m \geq 2$.

\section{ACKNOWLEDGEMENTS}

The authors thank N. Kalton and the referee for their helpful suggestions.

\section{REFERENCES}

[1] D. Achour and L. Mezrag, On the Cohen strongly p-summing multilinear operators, J. Math. Anal. Appl. 327 (2007), 550-563. MR 2277434(2007j:46076)

[2] R. Alencar and M. Matos. Some classes of multilinear mappings between Banach spaces, Publ. Dep. Analisis Mat. Univ. Complut. Madrid 12 (1989).

[3] R. Aron, M. Lacruz, R. Ryan and A. Tonge, The generalized Rademacher functions, Note Mat. 12 (1992), 15-25. MR.1258560 (95f:46029)

[4] F. Bombal, D. Pérez-García and I. Villanueva, Multilinear extensions of Grothendieck's theorem, Quart J. Math. 55 (2004), 441-450. MR.2104683 (2005i:47032)

[5] G. Botelho, Cotype and absolutely summing multilinear mappings and homogeneous polynomials, Proc. Roy. Irish Acad Sect. A 97 (1997), 145-153. MR1645283 (99i:46006)

[6] G. Botelho, Ideals of polynomials generated by weakly compact operators, Note Mat. 25 (2005/2006), 69-102. MR2220454 (2006m:46055)

[7] G. Botelho, H.-A. Braunss, H. Junek and D. Pellegrino, Holomorphy types and ideals of multilinear mappings, Studia Math. 177 (2006), 43-65. MR 2283707(2008a:46046)

[8] G. Botelho, H.-A. Braunss, H. Junek and D. Pellegrino, Inclusions and coincidences for multiple summing multilinear mappings, Proc. Amer. Math. Soc. 137 (2009), 991-1000. MR 2457439

[9] G. Botelho and D. Pellegrino, Scalar-valued dominated polynomials on Banach spaces, Proc. Amer. Math. Soc. 134 (2006), 1743-1751. MR2204287 (2006i:46063)

[10] G. Botelho and D. Pellegrino, Absolutely summing linear operators into spaces with no finite cotype, Bull. Belg. Math. Soc. Simon Stevin 16 (2009), 373-378. 
[11] G. Botelho, D. Pellegrino and P. Rueda, Pietsch's factorization theorem for dominated polynomials, J. Funct. Anal. 243 (2007), 257-269. MR2291438 (2007k:46080)

[12] G. Botelho, D. Pellegrino and P. Rueda, On composition ideals of multilinear mappings and homogeneous polynomials, Publ. Res. Inst. Math. Sci. 43 (2007), 1139-1155. MR2389796 (2008m:46089)

[13] G. Botelho, D. Pellegrino and P. Rueda, Summability and estimates for polynomials and multilinear mappings, Indag. Math. (N.S.) 19 (2008), 23-31. MR2466391

[14] E. Çaliskan and D. Pellegrino, On the multilinear generalizations of the concept of absolutely summing operators, Rocky Mountain J. Math. 37 (2007), 1137-1154. MR2360288 (2008k:46129)

[15] D. Carando and V. Dimant. On summability of bilinear operators, Math. Nachr. 259 (2003), 3-11. MR2009332(2004i:47037)

[16] Y. S. Choi, S. G. Kim, Y. Meléndez and A. Tonge. Estimates for absolutely summing norms of polynomials and multilinear maps, Quarterly J. Math. 52 (2001), 1-12. MR.1820898 (2002g:46025)

[17] R. Cilia and J. Gutiérrez, Dominated, diagonal polynomials on $\ell_{p}$ spaces, Arch. Math. (Basel) 84 (2005), 421-431. MR2139545 (2005m:46071)

[18] A. Defant and K. Floret, Tensor norms and operator ideals, North-Holland Mathematics Studies, 176, North-Holland, 1993. MR,1209438(94e:46130)

[19] A. Defant, D. García, M. Maestre and D. Pérez-García, Bohr's strip for vector valued Dirichlet series, Math. Ann. 342 (2008), 533-555. MR.2430989

[20] A. Defant and D. Pérez-García, A tensor norm preserving unconditionality for $\mathcal{L}_{p}$-spaces, Trans. Amer. Math. Soc. 360 (2008), 3287-3306. MR2379797(2008m:46091)

[21] A. Defant and P. Sevilla-Peris, A new multilinear insight on Littlewood's 4/3-inequality, J. Funct. Anal. 256 (2009), 1642-1664.

[22] J. Diestel, H. Jarchow and A. Tonge, Absolutely summing operators, Cambridge University Press, 1995. MR 1342297 (96i:46001)

[23] V. Dimant, Strongly p-summing multilinear operators, J. Math. Anal. Appl. 278 (2003), 182-193. MR.1963473 (2003m:47031)

[24] S. Dineen, Complex analysis on infinite dimensional spaces, Springer-Verlag, London, 1999. MR.1705327 (2001a:46043)

[25] K. Floret and M. C. Matos, Application of a Khintchine inequality to holomorphic mappings, Math. Nachr. 176 (1995), 65-72. MR1361126 (96j:46042)

[26] S. Geiss, Ideale multilinearer Abbildungen, Diplomarbeit, 1985.

[27] H. Jarchow, C. Palazuelos, D. Pérez-García and I. Villanueva, Hahn-Banach extension of multilinear forms and summability, J. Math. Anal. Appl. 336 (2007), 1161-1177. MR2353008 (2008k:46132)

[28] H. Junek, M. C. Matos and D. Pellegrino, Inclusion theorems for absolutely summing holomorphic mappings, Proc. Amer. Math. Soc. 136 (2008), 3983-3991. MR2425739

[29] M. C. Matos, On multilinear mappings of nuclear type, Rev. Mat. Univ. Complut. Madrid 6 (1993), 61-81. MR.1245025 (94j:46021)

[30] M. C. Matos. Absolutely summing holomorphic mappings, An. Acad. Brasil. Ciênc. 68 (1996), 1-13. MR1752625 (2001c:46086)

[31] M. C. Matos. Fully absolutely summing and Hilbert-Schmidt multilinear mappings, Collectanea Math. 54 (2003), 111-136. MR1995136 (2004e:46052)

[32] M. C. Matos and D. Pellegrino. Fully summing mappings between Banach spaces, Studia Math. 178 (2007), 47-61. MR2282489 (2007j:46117)

[33] Y. Meléndez and A. Tonge, Polynomials and the Pietsch domination theorem, Proc. Roy. Irish Acad. Sect. A 99 (1999), 195-212. MR1881812 (2003e:46026)

[34] J. Mujica, Complex analysis in Banach spaces, North-Holland Mathematics Studies, 120, North-Holland, 1986. MR842435 (88d:46084)

[35] D. Pellegrino, Cotype and absolutely summing homogeneous polynomials in $\mathcal{L}_{p}$ spaces, Studia Math. 157 (2003), 121-131. MR1980709 (2004f:46019)

[36] D. Pellegrino and M. Souza, Fully and strongly almost summing multilinear mappings, Rocky Mountain J. Math. 36 (2006), 683-698. MR2234827 (2007b:46068)

[37] D. Pérez-García, The inclusion theorem for multiple summing operators, Studia Math. 165 (2004), 275-290. MR2110152 (2005i:47107) 
[38] D. Pérez-García, Comparing different classes of absolutely summing multilinear operators, Arch. Math. (Basel) 85 (2005), 258-267. MR2172384 (2006j:46055)

[39] D. Pérez-García and I. Villanueva, A composition theorem for multiple summing operators, Monatsh. Math. 146 (2005), 257-261. MR2184227 (2006g:46072)

[40] D. Pérez-García, The trace class is a Q-algebra, Ann. Acad. Sci. Fenn. Math. 31 (2006), 287-295. MR2248816(2007j:46116)

[41] D. Pérez-García and I. Villanueva, Multiple summing operators on Banach spaces, J. Math. Anal. Appl. 285 (2003), 86-96. MR2000141 (2004m:47039)

[42] D. Pérez-García, M. M. Wolf, C. Palazuelos, I. Villanueva and M. Junge, Unbounded violation of tripartite Bell inequalities, Commun. Math. Phys. 279 (2008), 455-486. MR.2383595 $(2008 \mathrm{~m}: 81018)$

[43] A. Pietsch. Ideals of multilinear functionals (designs of a theory), Proceedings of the Second International Conference on Operator Algebras, Ideals and Their Applications in Theoretical Physics, 185-199, Teubner-Texte Math., 67, Teubner, Leipzig, 1984. MR763541

[44] D. Popa, Reverse inclusions for multiple summing operators, J. Math. Anal. Appl. 350 (2009), 360-368. MR2476921

[45] I. Zalduendo, An estimate for multilinear forms on $\ell_{p}$ spaces, Proc. Roy. Irish. Acad. Sect. A 93 (1993), 137-142. MR1241848 (94j:46027)

Faculdade de Matemática, Universidade Federal de Uberlândia, 38.400-902, UberLÂNDIA, BRAZIL

E-mail address: botelho@ufu.br

Departamento de Matemática, Universidade Federal da Paraíba, 58.051-900, João Pessoa, BraziL

E-mail address: dmpellegrino@gmail.com

Departamento de Análisis Matemático, Universidad de Valencia, 46.100 Burjasot, VALENCIA, SPAin

E-mail address: pilar.rueda@uv.es 